\title{
Task Readiness Impedance in Human Arm Movements for Virtual Ball-Catching Task
}

\author{
Yoshiyuki Tanaka, Toshio Tsuji and Makoto Kaneko \\ Graduate School of Engineering, Hiroshima University \\ Higashi-hiroshima, Hiroshima 739-8527, JAPAN \\ E-mail: ytanaka@bsys.hiroshima-u.ac.jp \\ URL: http://www.bsys.hiroshima-u.ac.jp
}

\begin{abstract}
The dynamic characteristics of human upper extremities can be modeled with mechanical impedance. Although many studies have been reported on the human hand impedance properties in static tasks with arm posture maintained, there are no reports for human arm movements in dynamic tasks including interactions with environments. The human impedance should be regulated before motion in some tasks. In the ball-catching task, for instance, a human must be regulated his/her hand impedance to catch a ball before the hand contacts the ball. This paper examines human impedance in preparation for task operations, i.e., "task-readiness impedance," and proposes a method of measuring hand impedance during tasks using a virtual-reality technique. Experiments are then conducted to examine task-readiness impedance as well as the virtual trajectory in a virtual ball-catching task.
\end{abstract}

\section{INTRODUCTION}

A human performs a variety of skillful movements by adjusting dynamic characteristics of his/her musculoskeletal system according to tasks. In the ball-catching task by the human upper extrimity, for instance, a human player might miss the ball when he makes his arm stiffen beyond necessity for the task because of the large contact force exerted on the hand from the ball. On the other hand, when the player's arm is too compliant, he also might miss because he cannot generate hand force enough to absorb the ball motion. Thus, the player has to regulate mechanical properties of his arm to catch a ball according to task conditions such as ball speed, weight, size and so on. In general, such mechanical characteristics of human hand can be described with the mechanical impedance parameters; stiffness, viscosity, and inertia.

On the other hand, many experimental studies on human hand impedance have been reported in multi-joint arm movements. For example, Mussa-Ivaldi et al. [1] pioneered the measurement of human hand impedance, and examined hand stiffness in stable arm posture. They found that the hand stiffness strongly depends on the arm posture and that a human can change the size of a stiffness ellipse, although he/she can change neither the orientation nor the shape of it. Also, Dolan et al. [2] and Tsuji et al. [3][4] investigated not only hand stiffness but also viscosity and inertia, and verified a qualitative analogy between hand stiffness and viscosity. Tsuji et al.[5] also showed that the human hand viscoelastisity is widely changed with respect to the muscle activation level during isometric contraction in the upper limb. Moreover, Gomi and Kawato [6] have estimated the hand stiffness during a reaching movement. They reported that the hand stiffness is changed considerably during reaching movements comparing to the one during maintained arm posture. In addition, the virtual trajectory was calculated using the estimated hand impedance parameters.

There have been many researches on the human hand impedance during maintained arm posture, and also during reaching movements in the free space. These study works, however, do not discuss the adaptation mechanism of human hand impedance according to task conditions, such as the interaction with environments, the goal of task, and so on. To the contrary, Bennett et al.[8] described the dynamic properties of human movements with an open loop transfer function, and analyzed the gain and the phase characteristics in catching a falling ball by human subjects. They also examined the change of transfer characteristics caused by stretch reflex and voluntary muscle activation under various conditions. However, this research focused on hand impedance only during the uniarticular movements of the elbow joint, but not the multi-joints arm movements. Besides, the change of human hand impedance does not mentioned clearly although the ball-catching task belongs to dynamic movements.

The present paper aims to investigate human hand impedance in the multi-joint arm movements during dynamic tasks considering the interaction of a human with his/her environments. Measuring human hand impedance under such task conditions will allow not only to clarify the regulation mechanism of human impedance according to dynamic tasks but also to implement the skill of experts into robot motion control. This paper is organized as follows: Section II proposes the estimation method of task-readiness impedance. Then, a virtual ball-catching task is taken up as an example of dynamic tasks in Section III, and the regulation ability of human impedance for the task is investigated through a set of the experimental and the simulation results in Section IV.

\section{TASK-READINESS IMPEDANCE}

\section{A. Method of Impedance Measurement}

Let us consider multi-joint movements by the human upper extremity in the $l$-dimensional task space. When the subject's end-point is displaced from its equilibrium by a small disturbance with a short duration as shown in Fig. II-B, dynamic characteristics of the hand can be expressed with an impedance model [3][4] as

$$
M_{e} \ddot{X}_{e}(t)+B_{e} \dot{X}_{e}(t)+K_{e}\left(X_{e}(t)-X_{v}(t)\right)=-F_{e}(t),
$$

where $F_{e}(t) \in \Re^{l}$ denotes the hand force applied to the environment; $X_{e}(t) \in \Re^{l}$ the hand position; $X_{v}(t) \in \Re^{l}$ the virtual 
trajectory; and $M_{e}, B_{e}$, and $K_{e} \in \Re^{l \times l}$ represent hand inertia, viscosity and stiffness, respectively. Assuming that $X_{v}(t)$ is constant, the following equation of hand dynamics can be derived from (1):

$$
M_{e} d \ddot{X}(t)+B_{e} d \dot{X}(t)+K_{e} d X(t)=-d F_{e}(t)
$$

where $d X(t)=X_{e}(t)-X_{e}\left(t_{0}\right), d F(t)=F_{e}(t)-F_{e}\left(t_{0}\right)$, and $t_{0}$ denotes the time when the disturbance is applied to the hand. In this model, the hand impedance matrices can be estimated from the measured hand position $X_{e}(t)$ and the hand force $F_{e}(t)$, induced by the external disturbance, with the least squares method [4].

In dynamic tasks, however, $X_{v}(t)$ usually changes and the measurable variables are only hand position $\left(X_{e}(t), \dot{X}_{e}(t), \ddot{X}_{e}(t)\right)$ and hand force $\left(F_{e}(t)\right)$. Therefore, $M_{e}, B_{e}, K_{e}$, and $X_{v}(t)$ cannot be uniquely determined. Moreover, the hand impedance should be regarded as a time-varying factor since impedance parameters change according to the arm posture and the muscle contraction level during dynamic tasks [6]. It is thus very difficult to estimate the hand impedance during dynamic tasks.

A human needs to regulate his/her hand impedance before motion in some target tasks. In the ball-catching task, for instance, a player should adjust his hand impedance before catching the ball according to the ball velocity as well as its physical properties; otherwise, it would be too late to prepare for the task. This suggests that the skilled player performs the target task successfully by regulating his hand impedance to desired properties in preparation for the target task before motions based on prior experience.

For the virtual trajectory, $X_{v}(t)$, can be regarded as a constant profile before motion, the hand impedance parameters can be estimated by using (2) in this phase. This paper focuses on such hand impedance in the preliminary phase, so-called taskreadiness impedance. Although task-readiness impedance differs from human impedance in dynamic motion, a human regulates his/her task-readiness impedance according to the given task before motion. Thus, analyzing task-readiness impedance may clarify the function of human impedance, such as learning ability and the adaptation mechanism of human impedance properties according to tasks.

\section{B. Virtual Trajectory}

In the virtual trajectory control hypothesis [7], hand motion is created by changes of the virtual trajectory, the interaction force with environments, and the hand impedance properties during tasks. Accordingly, in the ball-catching task, the hand of a player will follow the virtual trajectory if he missed the ball and the interaction force is not applied to the hand. In such a situation, the virtual trajectory can be calculated by using (1) as follows:

$$
X_{v}(t)=K_{e}^{-1}\left(F_{e}(t)+M_{e} \ddot{X}_{e}(t)+B_{e} \dot{X}_{e}(t)\right)+X_{e}(t),
$$

where $M_{e}, B_{e}$, and $K_{e}$ represent the task-readiness impedance parameters.

The virtual trajectory obtained by (3) can represent hand movements until just after motion, although the hand impedance

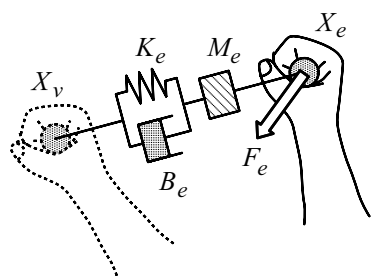

Fig.1. Schematic description of hand impedance

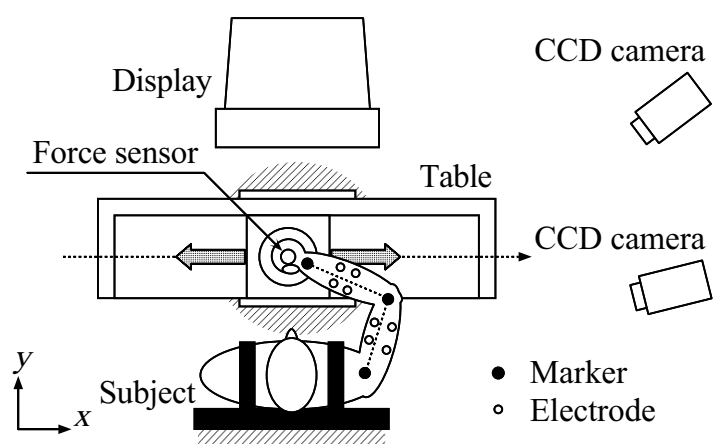

Fig.2. Experimantal apparatus for measuring task-readiness impedance in the virtual task

may start to change from just before motion. However, the difference between hand impedance just after motion and taskreadiness impedance will be so small that the virtual trajectory derived by (3) will be almost equal to the true virtual trajectory just after the motion begins.

Consequently, task-readiness impedance and the virtual trajectory can be estimated by providing the enforced displacement to move the hand in the miss-catching operation. However, it is not so easy to implement such an instantaneous operation during dynamic motions in a real ball-catching task. Therefore, a virtual ball-catching task is demonstrated in virtual space by using virtual reality technology in this paper.

\section{IMPEDANCE MEASURMENTS IN THE VIRTUAL TASK}

\section{A. Experimant Apparatus}

Figure 2 shows the experimental apparatus developed in this study. The system is composed of a robot that provides the interaction force between the computed virtual ball and the racket to a human subject, a computer for robot motion control as well as signal processing, and a display to indicate the task information to the subject.

The subject is required to hit the virtual ball instead of hitting an actual ball by operating the handle attached to the robot on the basis of the visual information provided on the bio-feedback display, while the robot displays interaction force to the subject at hitting the ball. The robot in the developed system is constructed with a linear motor table (Nippon Thompson Co., Ltd.; maximum driving force 10 [kgf]; stroke length 400 [mm]; encoder resolution $2[\mu \mathrm{m}]$ ), which is impedance-controlled so that the virtual interaction force between the virtual ball and the racket handle can be displayed to the subject. A six-axis force sensor (B.L. Autotech Co., Ltd.; resolution: translational force on $x$ - and $y$-axes $5 \times 10^{-3}[\mathrm{~N}]$, on $z$-axis $15 \times 10^{-2}[\mathrm{~N}]$, torque 


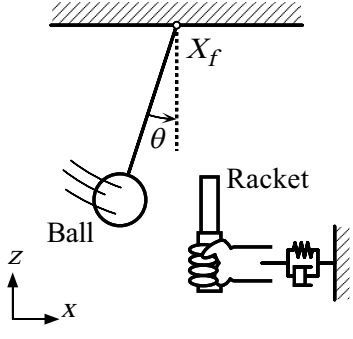

(a)

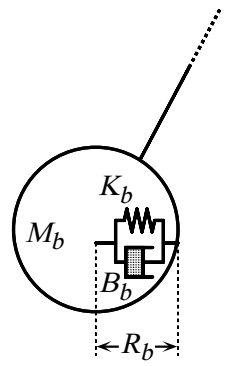

(b)
Fig.3. A model of the virtual ball-catching task

$\left.3 \times 10^{-3}[\mathrm{Nm}]\right)$ is attached at the base of the handle to measure the operating force by the subject. Then the developed system can estimate human hand impedance during maintained arm posture with accuracy [9].

A human can change the hand impedance properties by adjusting the muscle contraction level as well as his/her arm posture [5]. To investigate a mechanism of human impedance regulation, the surface EMG signals in the training are measured from the flexor (flexor carpi radialis: FCR) and the extensor (extensor ulnaris: ECU) in the wrist joint, the flexor (biceps brachii: $\mathrm{BB}$ ) and extensor (triceps brachii: $\mathrm{TB}$ ) in the elbow joint, and the flexors (pectoralis major: PM, deltoideus anterior: DA) and extensors (teres major: TM, deltoideus posterior: DP) in the shoulder joint. The sampling rate for measuring hand movements and EMG signals was set at $1[\mathrm{kHz}]$ in the experiments. Also, the stereo video camera system with two CCD cameras (Quick MAG: Oh-yoh Keisoku Kenkyusho, sampling rate: 60 $[\mathrm{Hz}])$ is utilized to observe subject's arm posture from the detected three-dimensional positions of color markers attached to subject's body.

\section{B. Virtual Ball-catching Task}

Figure 3(a) shows an overview of a virtual ball-catching task in the one dimensional task space $(l=1)$, in which the ball is hung from the ceiling at $X_{f}$ by a rigid pendulum with length $L$ and angle $\theta$. The initial position of the hand is set at the origin of the task space. The ball is approximated with a viscoelastic model as shown in Fig. 3(b), and the racket is regareded as a rigid body.

The interaction force $F_{\text {int }}$ between the ball and the racket is calculated from the relative position $X_{r}(t)\left(=X_{o}(t)-X_{e}(t)\right)$ by

$$
\begin{gathered}
F_{\text {int }}(t)=\left\{\begin{array}{cc}
B_{b} d \dot{X}_{b}(t)+K_{b} d X_{b}(t) & \left(\left|X_{r}(t)\right| \leq R_{b}\right) \\
0 & \left(\left|X_{r}(t)\right|>R_{b}\right)
\end{array}\right. \\
d X_{b}(t)=X_{r}(t)-R_{b} n \\
n= \begin{cases}\frac{X_{r}(t)}{\left|X_{r}(t)\right|} & \left(X_{r}(t) \neq 0\right) \\
0 & \left(X_{r}(t)=0\right)\end{cases}
\end{gathered}
$$

where $B_{b}$ and $K_{b}$ represent viscoelastic properties of the ball with weight $M_{b}$ and radius $R_{b}$; and $d X_{b}\left(=X_{r}-R_{b} n\right)$ represents a dent of the ball by the contact with the racket.

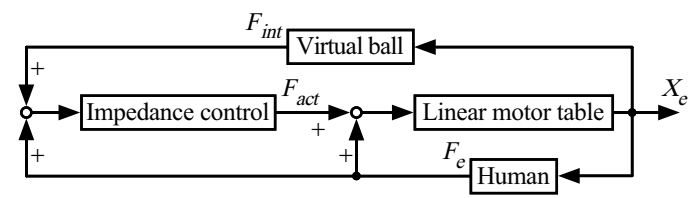

(a)

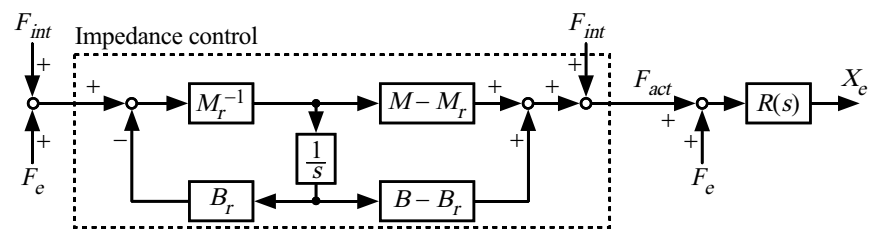

(b)

Fig.4. Impedance control system for the ball-catching task

On the other hand, the robotic table is under the impedance control [10] so that the racket position $X_{e}(t)$ follows

$$
M_{r} \ddot{X}_{e}(t)+B_{r} \dot{X}_{e}(t)=F_{\text {int }}(t)+F_{e}(t),
$$

where $M_{r}, B_{r}$ denote the desired inertia and viscosity of the robot. Fig. 4(a) shows a block diagram of the impedancecontrolled robot, where $F_{a c t}$ expresses a control input to the robot. Designing dynamics of the robot $R(s)$ by

$$
R(s)=\frac{1}{M s^{2}+B s},
$$

the impedance control is expressed as shown in Fig. 4 (b), where $M, B$ denote inertia and viscosity of the table, respectively. Here, the employed robot is with $M=4.7[\mathrm{~kg}]$ and $B=47$ $[\mathrm{Ns} / \mathrm{m}]$.

\section{Human Impedance in BALl-CATChing TASK}

\section{A. Human Arm Movemets}

Experiments were carried out by the unskilled subjects (Subjects A, B), who had never trained for the virtual ball-catching, and the skilled subjects (Subjects C, D) under $X_{f}=[0.3,2.1]$ [m], $L=2.1$ [m], and $\theta_{0}=-25$ [deg.]. The model parameters of a virtual ball defined in (4) were set as $M_{b}=0.5[\mathrm{~kg}]$, $B_{b}=20[\mathrm{Ns} / \mathrm{m}], K_{b}=2000[\mathrm{~N} / \mathrm{m}]$, and $R_{b}=0.03[\mathrm{~m}]$. The robot was controlled with $M_{r}=1[\mathrm{~kg}]$ and $B_{r}=20[\mathrm{Ns} / \mathrm{m}]$, and the ball started to move 1 [s] after a starting signal was displayed to the subject.

In the ball-catching task, a subject is required to control an interaction force as small as possible, otherwise the ball would rebound from the racket. Therefore, skill levels for the target task of subjects were evaluated with the maximum interaction force between the ball and the racket, $F_{i n t}^{\max }$, and the contact time of the ball and the racket, $t_{i n t}$, defined by

$$
\begin{gathered}
F_{\text {int }}^{\max }=\max _{0 \leq t \leq t_{e}} F_{\text {int }}(t), \\
t_{\text {int }}=\sum_{t=0}^{t_{e}} u(t) \Delta t, \\
u(t)= \begin{cases}1 & \left(F_{\text {int }}(t) \neq 0\right) \\
0 & \left(F_{\text {int }}(t)=0\right),\end{cases}
\end{gathered}
$$




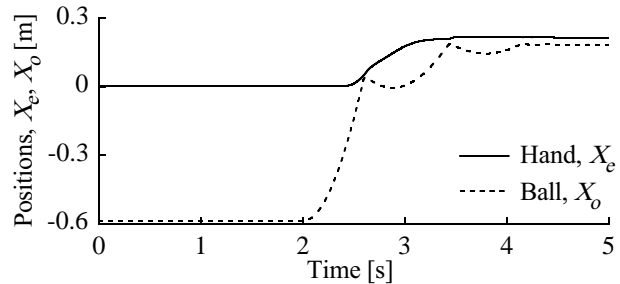

(a) Hand and ball positions

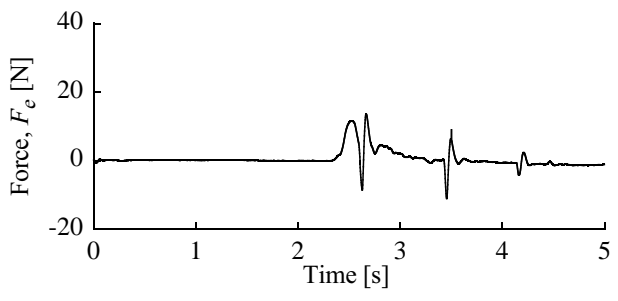

(b) Hand force

Fig.5. An example of experimental results by the unskilled subject $\mathrm{A}\left(F_{\text {int }}^{\max }=\right.$ $\left.38.09[\mathrm{~N}], t_{\text {int }}=0.69[\mathrm{~s}]\right)$

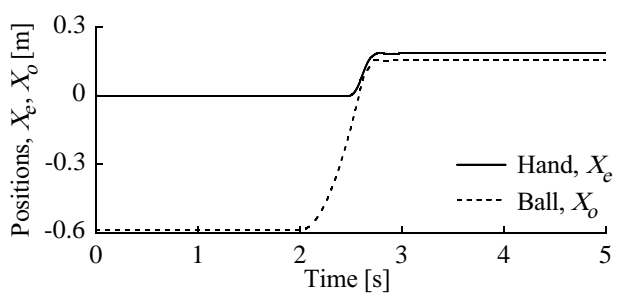

(a) Hand and ball positions

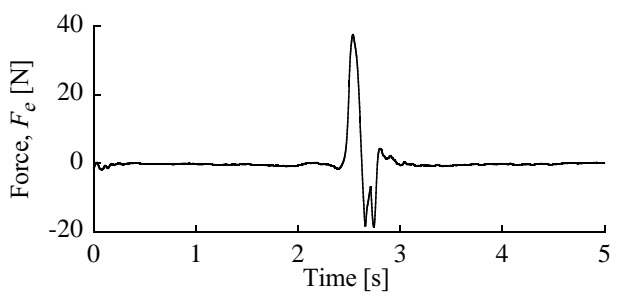

(b) Hand force

Fig.6. An example of experimental results by the skilled subject $\mathrm{C}\left(F_{\text {int }}^{\max }=\right.$ $\left.19.25[\mathrm{~N}], t_{\text {int }}=2.20[\mathrm{~s}]\right)$

where the measurement time in each trial was set at $t_{e}=5[\mathrm{~s}]$ and the sampling time at $\Delta t=1[\mathrm{~ms}]$. In the experiment, a subject was asked to minimize $F_{\text {int }}^{\max }$ and to maximize $t_{\text {int }}$.

Subjects were asked to perform the target task 120 times continuously during which the following five operations were executed in random order to measure human hand impedance: (I) measurement in normal catching task; (II) measurement during stable phase before motion; (III) measurement of task-readiness impedance in the preliminary phase; (IV) measurement of hand impedance after motion; and (V) estimation of the virtual trajectory when the subject misses the ball. The miss-catching operation is artificially conducted without preliminaries for a human operator during iterative trials, in which the subject does not feel any interaction force in contacting with the ball so that he/she cannot strike the ball. In the experiments, the subjects were instructed to maintain their arm postures until just before motion.
TABLE I

MAXIMUM INTERACTION FORCE AND CONTACTING TIME

\begin{tabular}{c|c||c|c}
\hline & & $F_{\text {int }}^{\max }[\mathrm{N}]$ & $t_{\text {int }}[\mathrm{s}]$ \\
\hline \hline \multirow{4}{*}{ Subjects } & $\mathrm{A}$ & $32.91 \pm 3.15$ & $1.16 \pm 0.45$ \\
\cline { 2 - 4 } & $\mathrm{B}$ & $32.39 \pm 3.89$ & $1.75 \pm 0.24$ \\
\cline { 2 - 4 } & $\mathrm{C}$ & $25.15 \pm 6.83$ & $1.70 \pm 0.29$ \\
\cline { 2 - 4 } & $\mathrm{D}$ & $25.22 \pm 3.83$ & $1.73 \pm 0.30$ \\
\hline
\end{tabular}

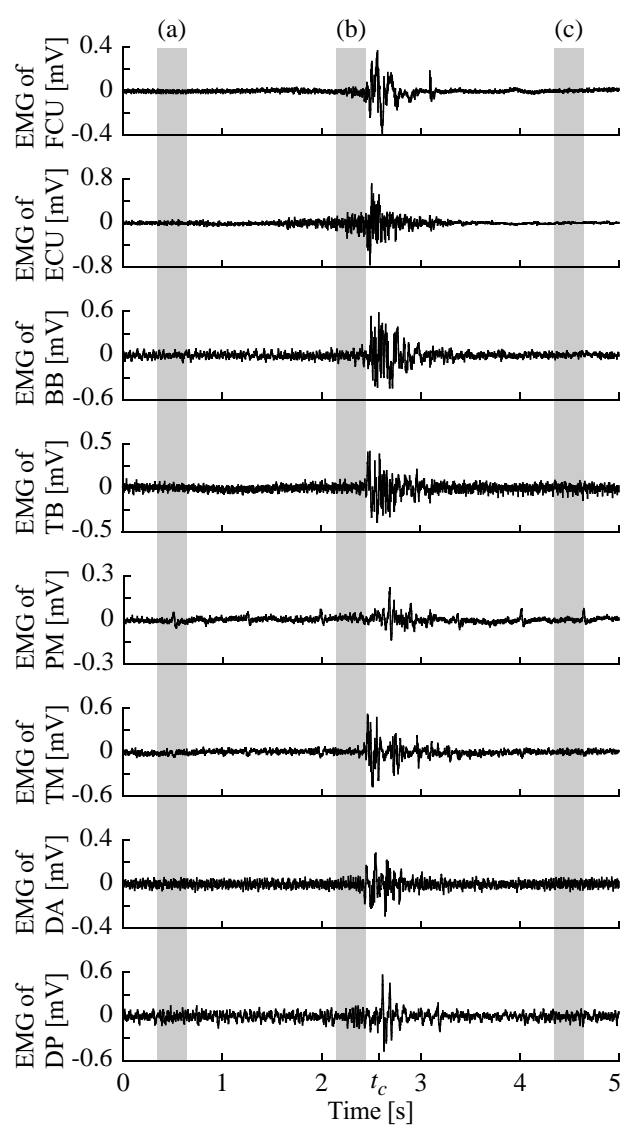

Fig.7. An example of the measured EMG signals (Subject C)

Figures 5 and 6 present the examples of experimental results by an unskilled subject (Subject A) and a skilled subject (Subject $\mathrm{C}$ ). The solid lines in the figure (a) represent the hand position of each subject, and the dotted lines indicate the ball position; the figure (b) illustrates the time profile of the subject's hand force during the task. The unskilled subject did not catch the ball, so the ball rebounds off the racket as shown in Fig. 5. In contrast, the skilled subject completes the target task by adjusting his hand velocity according to the ball motion. It can also be observed that the hand force applied by Subject $\mathrm{A}$ is much smaller than that applied by Subject C.

Table I shows the mean values of $F_{i n t}^{\max }$ and $t_{\text {int }}$ with standard deviations for a set of 120 trials by the subjects. Subjects C and $\mathrm{D}$ generated less large hand force for catching the ball with longer contact period than Subjects A and B. These results indicate that Subjects $C$ and $D$ have greater skill in the target task than do Subjects A and B. 


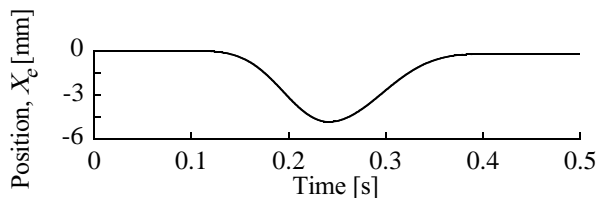

(a) Hand position

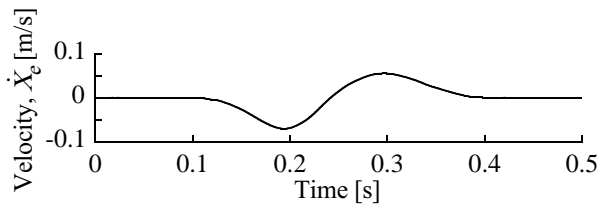

(b) Hand velocity

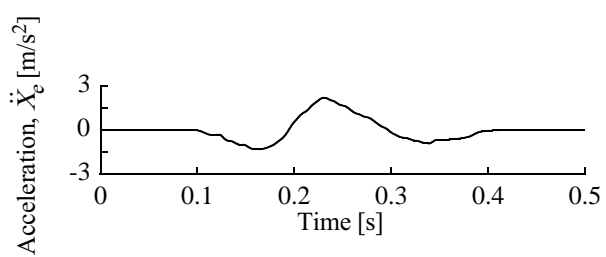

(c) Hand acceleration

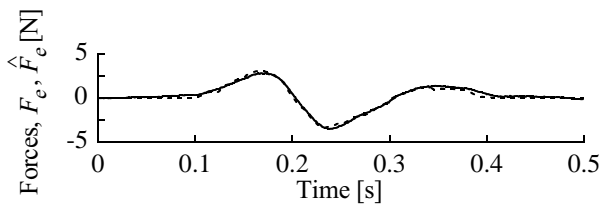

(d) Measured and estimated hand force

Fig.8. An example of measured signals for task-readiness impedance measurement (Subject C)

Figure 7 shows an example of EMG signals of the skilled subject during the task for 5 [s] measured from 1 [s] before a starting signal appears on the display, in which the racket contacts the ball at $t_{c}=2.6[\mathrm{~s}]$. It can be supposed that the subject contracts his arm muscles to prepare for the target task before the racket contacts the ball. Since the subject began to activate his arm muscle from $t=2.5[\mathrm{~s}]$, the external disturbances to the subject's hand for Operations II, III, and IV were induced at $t_{0}=0.5,2.3,0.45$ [s], respectively. Here, Operation III was executed 0.3 [s] before the contact. Shaded zones (a), (b) and (c) in Fig. 7 express the measuring terms for the estimation of hand impedance parameters in each operation.

\section{B. Hand Impedance Properties}

Figure 8 illustrates an example of measured hand movements for estimating task-readiness impedance. Figures (a), (b), and (c) express the time history of hand position $X_{e}(t)$, hand velocity $\dot{X}_{e}(t)$, and hand acceleration $\ddot{X}_{e}(t)$ caused by the external disturbance from the top in order. Also, the solid and the dotted lines in Fig. 8 (d) represent the measured hand force and the estimated hand force that is calculated by using (2) with the measured hand movements and the estimated hand-impedance parameters $\left(K_{e}, B_{e}, M_{e}\right)$. Figure $8(\mathrm{~d})$ demonstrates that hand impedance was estimated accurately because the solid line almost coincides with the dotted one.

Table II lists the mean values of the estimated impedance for each of the five operations with standard deviations and the correlation coefficients $\rho$ between the measured and the estimated
TABLE II

MEASURED HUMAN HAND IMPEDANCE PARAMETERS DURING THE BALL-CATCHING TASK

(a) During maintenance of the stable posture

\begin{tabular}{c|c||c|c|c|c}
\hline & & $K_{e}[\mathrm{~N} / \mathrm{m}]$ & $B_{e}[\mathrm{Ns} / \mathrm{m}]$ & $M_{e}[\mathrm{~kg}]$ & $\rho$ \\
\hline \hline \multirow{5}{*}{ Subjects } & $\mathrm{A}$ & $61.24 \pm 35.80$ & $21.84 \pm 0.53$ & $1.12 \pm 0.10$ & $0.95 \pm 0.01$ \\
\cline { 2 - 6 } & $\mathrm{B}$ & $43.69 \pm 23.71$ & $12.37 \pm 1.66$ & $0.94 \pm 0.06$ & $0.92 \pm 0.01$ \\
\cline { 2 - 6 } & $\mathrm{C}$ & $64.90 \pm 33.18$ & $25.39 \pm 2.51$ & $1.74 \pm 0.10$ & $0.96 \pm 0.01$ \\
\cline { 2 - 6 } & $\mathrm{D}$ & $98.34 \pm 45.04$ & $22.65 \pm 3.53$ & $1.64 \pm 0.11$ & $0.93 \pm 0.02$ \\
\hline
\end{tabular}

(b) Before motion

\begin{tabular}{c|c||c|c|c|c}
\hline & & $K_{e}[\mathrm{~N} / \mathrm{m}]$ & $B_{e}[\mathrm{Ns} / \mathrm{m}]$ & $M_{e}[\mathrm{~kg}]$ & $\rho$ \\
\hline \hline \multirow{5}{*}{ Subjects } & A & $72.74 \pm 42.51$ & $25.22 \pm 2.29^{* * *}$ & $1.18 \pm 0.09$ & $0.97 \pm 0.01$ \\
\cline { 2 - 6 } & B & $85.78 \pm 47.10^{* * *}$ & $16.50 \pm 2.29^{* * *}$ & $1.00 \pm 0.11$ & $0.97 \pm 0.01$ \\
\cline { 2 - 6 } & $\mathrm{C}$ & $84.21 \pm 41.12$ & $29.34 \pm 4.36^{*}$ & $1.74 \pm 0.12$ & $0.96 \pm 0.01$ \\
\cline { 2 - 6 } & $\mathrm{D}$ & $165.35 \pm 81.41^{* *}$ & $21.04 \pm 4.37$ & $1.71 \pm 0.18$ & $0.96 \pm 0.01$ \\
\hline
\end{tabular}

(c) Task readiness

\begin{tabular}{c|c||c|c|c|c}
\hline & & $K_{e}[\mathrm{~N} / \mathrm{m}]$ & $B_{e}[\mathrm{Ns} / \mathrm{m}]$ & $M_{e}[\mathrm{~kg}]$ & $\rho$ \\
\hline \hline \multirow{5}{*}{ Subjects } & $\mathrm{A}$ & $61.47 \pm 30.42$ & $24.26 \pm 3.12^{* *}$ & $1.25 \pm 0.14^{*}$ & $0.98 \pm 0.01$ \\
\cline { 2 - 6 } & $\mathrm{B}$ & $57.45 \pm 35.39$ & $17.56 \pm 3.02^{* * *}$ & $0.83 \pm 0.17^{* *}$ & $0.96 \pm 0.01$ \\
\cline { 2 - 6 } & $\mathrm{C}$ & $188.89 \pm 71.20^{* * *}$ & $28.07 \pm 2.37^{*}$ & $1.90 \pm 0.16^{* *}$ & $0.98 \pm 0.01$ \\
\cline { 2 - 6 } & $\mathrm{D}$ & $154.81 \pm 103.40$ & $22.27 \pm 2.95$ & $1.76 \pm 0.23$ & $0.96 \pm 0.01$ \\
\hline
\end{tabular}

(d) After motion

\begin{tabular}{c|c||c|c|c|c}
\hline & & $K_{e}[\mathrm{~N} / \mathrm{m}]$ & $B_{e}[\mathrm{Ns} / \mathrm{m}]$ & $M_{\mathrm{e}}[\mathrm{kg}]$ & $\rho$ \\
\hline \hline \multirow{5}{*}{ Subjects } & $\mathrm{A}$ & $117.27 \pm 67.62^{* *}$ & $24.98 \pm 3.42^{* * *}$ & $1.14 \pm 0.11$ & $0.99 \pm 0.01$ \\
\cline { 2 - 6 } & $\mathrm{B}$ & $82.02 \pm 43.70^{* * *}$ & $8.76 \pm 1.49^{* * *}$ & $0.81 \pm 0.10^{* * *}$ & $0.97 \pm 0.01$ \\
\cline { 2 - 6 } & $\mathrm{C}$ & $97.97 \pm 57.05$ & $23.94 \pm 4.53$ & $1.44 \pm 0.18^{* * *}$ & $0.97 \pm 0.01$ \\
\hline & $\mathrm{D}$ & $156.17 \pm 103.89$ & $10.04 \pm 2.88^{* * *}$ & $1.33 \pm 0.14^{* * *}$ & $0.98 \pm 0.01$ \\
\hline
\end{tabular}

hand force by using the least squares method with (2). The tables (a), (b), (c), and (d) describe the impedance properties in operations I, II, III, and IV, respectively. It should be noted that hand impedance was estimated with the same posture in operation I $\sim$ III. Asterisks $*$, **, and $* * *$ denote the estimated impedance parameters with significant differences under the significance level 5, 3,1\% for the hand-impedance parameters for a constant arm posture determined by the one-sided t-test.

All subjects increased hand stiffness before motion (Operation I), compared with maintaining the arm in a stable posture (Operation II). This indicates that a human begins regulating his/her impedance properties before executing the target task. In contrast, the estimated hand impedance did not differ significantly between Operations II and III, except for Subject C. This strongly suggests that a human begins regulating his/her hand impedance with enough time to prepare for tasks. Fig. 7 also reveals that the subjects contracted each muscle in the upper extremity from the beginning of the task $(t=0[s])$. The task-readiness stiffness of Subject $\mathrm{C}$ exceeded the stiffness before motion, because his muscular activity began to change from about $1.5[\mathrm{~s}]$. 


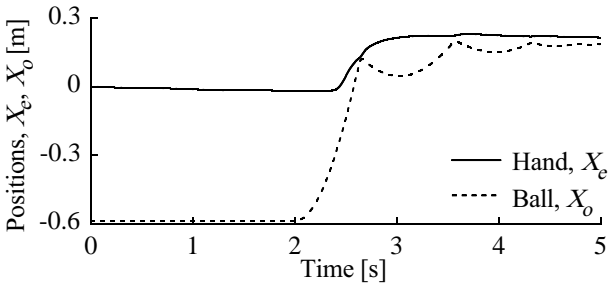

(a) Simulated hand and ball positions

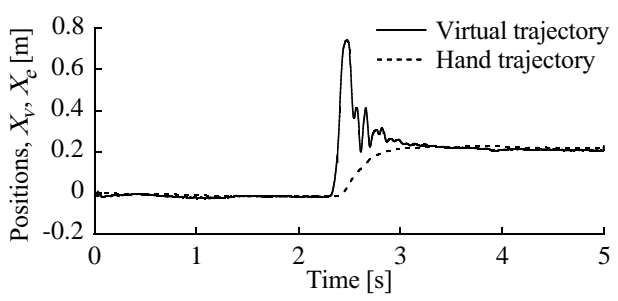

(b) Estimated virtual trajectory

Fig.9. Simulation results with the estimated task-readiness impedance of the unskilled subject A

\section{Simulations with Estimated Human Impedance}

Finally, we conducted computer simulations to reproduce human hand movements in the ball-catching task with the estimated task-readiness impedance and the virtual trajectory, assuming that the dynamic characteristics of the human hand follow the impedance model given in (1).

Figure 9 depicts the simulated results with the estimated taskreadiness impedance of the unskilled player (Subject A), corresponding to the experimental result in Fig. 5. Similarly, Fig. 10 is the simulated result using the task-readiness impedance of the skilled player (Subject C), corresponding to Fig. 6. The solid line in the figure (a) represents time profiles of the hand, and the dotted line, time profiles of the ball position. The solid (dotted) line in the figure (b) is the virtual (measured) hand trajectory. The ball in Fig. 10 smoothly converges to a stable point, while the one in Fig. 9 bounds off the hand. Dynamic properties of human movements during the task are thus reflected well in both the task-readiness impedance and the virtual trajectory.

The proposed methodology can potentially describe human skills, such as how humans perform tasks, in human impedance properties, which will be a key factor for developing a humanlike robot.

\section{CONCLUSION}

This paper has discussed how a human regulates his/her hand impedance properties for dynamic contact tasks through analyzing the task-readiness impedance and the virtual trajectory of the hand in a virtual ball-catching task. From a set of the experimental and the simulated results, we clarified the following points on human hand movements.

1. Human skill can be modeled with mechanical impedance properties.

2. Human impedance properties can be utilized to describe the skill level for tasks of human operators.

3. A human operator regulates his/her impedance properties appropriately in target tasks.

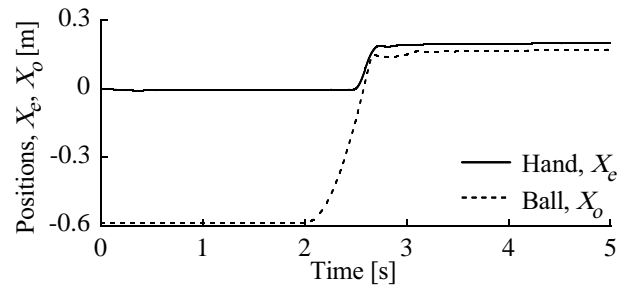

(a) Simulated hand and ball positions

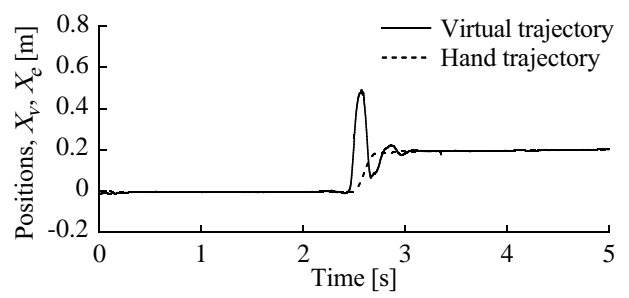

(b) Estimated virtual trajectory

Fig.10. Simulation results with the estimated task-readiness impedance of the skilled subject $\mathrm{C}$

Future research will investigate task-readiness impedance according to the impedance properties of an object and a robot as well as bio-feedback information during tasks. We will also examine how a human attains proficiency for a given task to clarify the regulation ability of task-readiness impedance in detail.

\section{Acknowledgments}

This research work was supported in part by a Grant-in-Aid for Scientific Research from the Japanese Ministry of Education, Science and Culture (15360226 and 14750188).

\section{REFERENCES}

[1] F. A. Mussa-Ivaldi, N. Hogan, and E. Bizzi: "Neural, Mechanical and Geometric Factors Subserving Arm in Humans," Journal of Neuroscience, vol.5, no. 10, pp. 2732-2743, 1985.

[2] J. M. Dolan, M. B. Friendman, and M. L. Nagarka: "Dynamics and Loaded Impedance Components in the Maintenance of Human Arm Posture," IEEE Transactions on Systems, Man, and Cybernetics, vol.23, no. 3, pp. 698-709, 1993.

[3] T. Tsuji, K. Goto, K. Ito and M. Nagamachi: "Estimation of Human Hand Impedance During Maintenance of Posture," Transactions of the Society of Instrument and Control Engineers, vol. 30, no. 3, pp. 319-328, 1994. (in Japanese)

[4] T. Tsuji, P. G. Morasso, K. Goto, and K. Ito: "Human Hand Impedance Characteristics During Maintained Posture," Biological Cybernetics, vol. 72, pp. 457-485, 1995

[5] T. Tsuji, M. Moritani, M. Kaneko and K. Ito: "An Analysis of Human Hand Impedance Characteristics : During Isometric Muscle Contractions," Transactions of the Society of Instrument and Control Engineers, vol. 32, no. 2, pp. 271-280, 1996. (in Japanese)

[6] H. Gomi, and M. Kawato: "Human Arm Stiffness and Equilibrium-Point Trajectory During Multi-Joint Movement," Biological Cybernetics, vol. 76, pp. 163-171, 1997.

[7] N. Hogan: "The Mechanics of Multi-Joint Posture and Movement Control," Biological Cybernetics, vol. 52, pp. 315-331, 1985.

[8] D. J. Bennett, M. Gorassini, and A. Prochazka: "Catching A Ball: Contributions of Intrinsic Muscle Stiffness, Reflexes, and Higher Order Responses," CAN. J. PHYSIOL. PHARMACOL, vol. 72, pp. 525-534, 1994.

[9] T. Tsuji, Y. Kanji, T. Kato, M. Kaneko, and S. Kawamura: "Impedance Training: Can We Regulate Our Hand Impedance Through Training?," Transactions of the Society of Instrument and Control Engineers, vol. 35, no. 10, pp. 1300-1306, 1999. (in Japanese)

[10] N. Hogan: "Impedance Control: An Approach to Manipulation: Parts I, II, III," ASME Journal of Dynamic Systems, Measurement, and Control, vol. 107, no. 1, pp. 1-24, 1985. 